\title{
THE MINIMUM DILATATION OF PSEUDO-ANOSOV 5-BRAIDS
}

\author{
JI-YOUNG HAM AND WON TAEK SONG
}

\begin{abstract}
The minimum dilatation of pseudo-Anosov 5-braids is shown to be the largest zero $\lambda_{5} \approx 1.72208$ of $x^{4}-x^{3}-x^{2}-x+1$ which is attained by $\sigma_{1} \sigma_{2} \sigma_{3} \sigma_{4} \sigma_{1} \sigma_{2}$.
\end{abstract}

\section{INTRODUCTION}

Let $f: D^{2} \rightarrow D^{2}$ be an orientation preserving disk homeomorphism which is the identity map on the boundary $\partial D^{2}$, and $\left\{p_{i}\right\} \subset \operatorname{int}\left(D^{2}\right)$ be a periodic orbit of $f$ (or more generally a finite set invariant under $f$ ). The points $p_{i}$ move under an isotopy from the identity map on $D^{2}$ to $f$. Their trajectory forms a geometric braid $\beta$, a collection of strands in $D^{2} \times[0,1]$ connecting $p_{i} \times 1$ to $f\left(p_{i}\right) \times 0$ (see Figure (1). The isotopy class of $\beta$ determines the homotopy class of $f$ relative to $\left\{p_{i}\right\} \cup \partial D^{2}$ and vice versa. An $n$-braid refers to the isotopy class of a geometric braid with $n$ strands. The set of $n$-braids forms the braid group $B_{n}$.

From now on we consider $f$ as a homeomorphism on a punctured sphere $f: \operatorname{int}\left(D^{2}\right)-$ $\left\{p_{i}\right\} \rightarrow \operatorname{int}\left(D^{2}\right)-\left\{p_{i}\right\}$. In particular by forgetting the boundary $\partial D^{2}$, we ignore Dehn twists along $\partial D^{2}$ which do not affect the dynamics of the braid $\beta$. In other words we consider an $n$-braid $\beta$ as a mapping class on a $(n+1)$-times punctured sphere with the (so called) boundary puncture fixed.

Topological entropy. The topological entropy $h_{T}(\beta)$ of the braid $\beta$ is defined to be the infimum topological entropy of the disk homeomorphisms representing $\beta$.

$$
h_{T}(\beta)=\inf _{g \simeq f} h_{T}(g)
$$

The topological entropy of a braid is a conjugacy invariant measuring the dynamical complexity of the braid. It is equal to the logarithm of the growth rate of the free group automorphism induced on $\pi_{1}\left(D^{2}-\left\{p_{i}\right\}\right)$. When $\beta$ is represented by a pseudo-Anosov homeomorphism $f$ with dilatation factor $\lambda_{f}=\lambda(f)$, we have $h_{T}(\beta)=\log \lambda_{f}$. In this case the dilatation $\lambda(\beta)$ of the braid is also given by $\lambda(\beta)=\lambda_{f}$.

Date: 14 June 2005; Revised 24 January 2006.

2000 Mathematics Subject Classification. Primary 37E30; Secondary 37B40, 57M60. 


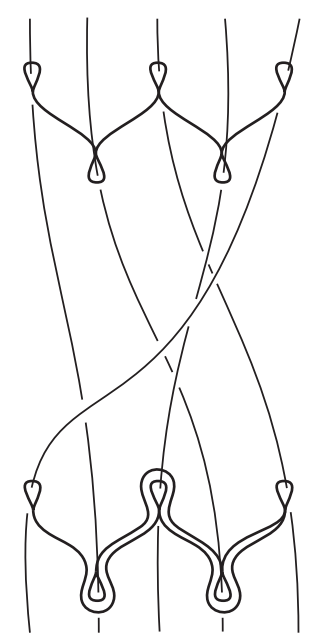

FiguRE 1 . This picture shows a pseudo-Anosov 5-braid $\sigma_{3} \sigma_{4} \sigma_{3} \sigma_{2} \sigma_{3} \sigma_{1}$ with its invariant train track. The train track $\tau \subset D^{2} \times 1$ on the top slides down in the complement of the braid to $f(\tau) \subset D^{2} \times 0$ on the bottom, where $f$ is the disk homeomorphism representing the braid.

If $f$ is homotopic to a periodic homeomorphism, the braid $\beta$ is called periodic. If there is a collection of disjoint sub-surfaces of $\operatorname{int}\left(D^{2}\right)-\left\{p_{i}\right\}$ with negative Euler characteristics which is homotopically invariant under $f$, the braid $\beta$ is called reducible. As we consider the dynamics of a periodic braid to be trivial, studying the dynamics of braids reduces to the maps on non-periodic irreducible components.

Pseudo-Anosov homeomorphism. By the Nielsen-Thurston classification of surface homeomorphisms [26, 3, 5, 9], a non-periodic irreducible braid is represented by a pseudoAnosov homeomorphism. A pseudo-Anosov homeomorphism has several nice extremal properties. It realizes the minimum topological entropy and the minimum quasi-conformality constant in its homotopy class. It also has the minimum number of periodic orbits for each period [6].

A surface homeomorphism $f: F \rightarrow F$ is called a pseudo-Anosov homeomorphism relative to a puncture set $\left\{p_{i}\right\} \subset F$ when the following conditions hold. First we need a singular flat metric on $F$ with a finite singularity set $\left\{q_{j}\right\}$ such that $\left\{p_{i}\right\} \subset\left\{q_{j}\right\}$. Each singularity $q_{j}$ has its cone-angle in $\left\{k \pi \mid k \in \mathbb{Z}_{>0}\right\}$. If a singularity has cone-angle $\pi$, it must be one of the puncture points $p_{i}$. Now the homeomorphism $f$ is required to preserve $\left\{q_{j}\right\}$ and to be locally affine (hyperbolic) on $F-\left\{q_{j}\right\}$ with a constant dilatation factor $\lambda_{f}>1$. In particular at a fixed point in $F-\left\{q_{j}\right\}$, the map $f$ is locally written as $\left[\begin{array}{cc}\lambda_{f} & 0 \\ 0 & \lambda_{f}^{-1}\end{array}\right]$. 
Thus roughly speaking, if a surface homeomorphism $f$ represents a non-periodic irreducible mapping class, then one can simplify $f$ by pulling tight it everywhere until it becomes linear almost everywhere in an appropriate sense.

The horizontal directions to which $f$ expands by the factor $\lambda_{f}$ integrate to form one invariant measured foliation $\mathcal{F}^{s}$. The vertical directions perpendicular to $\mathcal{F}^{s}$ form the other invariant measured foliation $\mathcal{F}^{u}$. From a singularity $q_{j}$ with cone-angle $k \pi, k$-many singular leaves of $\mathcal{F}^{s}$ emanate. In this case $q_{j}$ is called a $k$-prong singularity.

Note that in the above definition of pseudo-Anosov homeomorphism we can remove or add punctures keeping the same map $f: F \rightarrow F$. When $\left\{f^{j}(x)\right\}$ is a periodic orbit of nonpunctured points, puncturing at $\left\{f^{j}(x)\right\}$ refers to adding them to the puncture set $\left\{p_{i}\right\}$. Conversely when $\left\{f^{j}\left(p_{1}\right)\right\}$ is a periodic orbit of $k$-prong punctured singularities for $k>1$, capping-off them refers to removing them from the puncture set. For pseudo-Anosov braids, puncturing or capping-off corresponds to adding or removing some strands.

Let $\tilde{f}: \tilde{F} \rightarrow \tilde{F}$ be a lift of $f$ on a finite-fold cover $\tilde{F}$ of $F$ branched at some finite set of points invariant under $f$. Then by pulling back the flat metric on $F$ to $\tilde{F}$, the lift $\tilde{f}$ is also a pseudo-Anosov homeomorphism with the same dilatation factor $\lambda_{\tilde{f}}=\lambda_{f}$.

Train track representative. Using a Markov partition (or its associated train track representative), the flat metric and the pseudo-Anosov homeomorphism can be described quite concretely (see [10, Exposé 9] for the definition and see Figure 2 for an example). Let $\left\{R_{i}\right\}$ be a Markov partition for a pseudo-Anosov homeomorphism $f$. The transition matrix $M_{f}=\left(m_{i j}\right)$ is defined by that $f\left(R_{i}\right)$ crosses over $R_{j} m_{i j}$-many times.

The transition matrix $M_{f}$ is Perron-Frobenius: for some $k>0$ each entry of $M_{f}^{k}$ is strictly positive. In particular the largest eigenvalue of $M_{f}$ is real and has an eigenvector with strictly positive coordinates [21, Theorem 1.1 on p.1].

The widths $v_{i}$ and the heights $w_{i}$ of $R_{i}$ satisfy the following equations.

$$
\lambda_{f} v_{i}=\sum_{j} m_{i j} v_{j} \quad w_{j}=\sum_{i} m_{i j} w_{i} / \lambda_{f}
$$

In particular the dilatation factor $\lambda_{f}$ appear as the eigenvalue of $M_{f}$ of which the eigenvector has strictly positive coordinates.

We use train track representatives as a notational simplification for Markov partitions. As in Figure 2, each expanding edge of the invariant train track corresponds to a rectangle in the Markov partition. Once given the transition matrix of the graph map, it is easy to recover the heights and widths of the rectangles. 

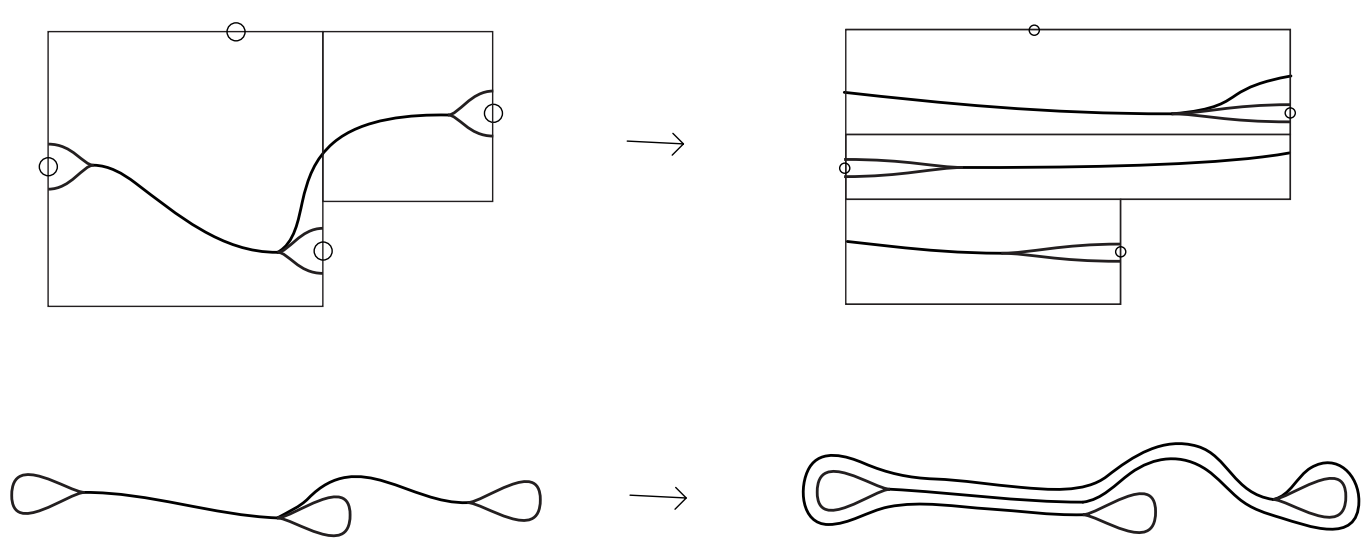

FiguRE 2. The boundary of the $L$-shaped region reads, counterclockwise from the puncture on the top, $a b b^{-1} c d d^{-1} e f f^{-1} g$ with $a c=g^{-1} e^{-1}$. After side-pairing, the $L$-shaped region becomes a 4 -times punctured sphere with a flat metric. The pseudo-Anosov homeomorphism maps each rectangle to a longer and thinner horizontal strip running over other rectangles. In the train track representative, each edge is assigned tangential and transverse measures coming from the width and the height of the corresponding rectangle.

Main question. Let us consider the set $\Lambda_{g, n}$ of the dilatation factors for pseudo-Anosov homeomorphisms on an $n$-times punctured genus- $g$ surface $F_{g, n}$.

$$
\Lambda_{g, n}=\left\{\lambda_{f} \mid f: F_{g, n} \rightarrow F_{g, n} \text { pseudo-Anosov homeomorphisms }\right\}
$$

As we can bound the number of rectangles in Markov partitions using the Euler characteristic of the punctured surface, $\Lambda_{g, n}$ consists of eigenvalues of Perron-Frobenius matrices with bounded dimension. In particular the set $\Lambda_{g, n}$ is discrete and has a minimum. Our current work is motivated by the following question.

Question 1.1. What is $\min \Lambda_{g, n}$ ?

The question asks to find the simplest pseudo-Anosov homeomorphism on the surface.

A pseudo-Anosov homeomorphism $f$ induces an isometry on the Teichmüller space equipped with the Teichmüller metric. The pair of invariant measured foliations $\left(\mathcal{F}^{s}, \mathcal{F}^{u}\right)$ determines a geodesic axis in the Teichmüller space on which $f$ acts as a translation by $\log \lambda_{f}$. The axis projects down to a closed geodesic in the moduli space, which is the quotient of the Teichmüller space by the action of the mapping class group. Conversely any closed geodesic in the moduli space represents the conjugacy class of some pseudo-Anosov 
mapping class. Therefore Question [1.1 can be rephrased as asking to find the shortest closed geodesic in the moduli space.

The hyperbolic volume of the mapping torus is another natural complexity measure for a pseudo-Anosov homeomorphism. We notice that a pseudo-Anosov homeomorphism with small dilatation tends to have the mapping torus with small hyperbolic volume and vice versa.

The question for the minimum volume of the hyperbolic mapping tori on a given surface seems to be much more difficult than Question [1.1, In [8] the minimum volume for orientable cusped hyperbolic 3-manifolds is computed. An extensive use of computer programs is involved in its proof. In this paper we also use a computer program for the proof of the main theorem, but the algorithm and the actual code is much simpler than those of [8].

Related results. The question asking for the minimum dilatation of pseudo-Anosov homeomorphisms on a given surface still remains largely unanswered since after the NielsenThurston classification of surface homeomorphisms. The existence of Markov partition [10. Exposé 10] for a pseudo-Anosov homeomorphism implies that a dilatation should appear as the largest eigenvalue of a Perron-Frobenius matrix of bounded dimension, hence in particular should be an algebraic integer. However, it is not clear how the restriction that the symbolic dynamical system dictated by a Perron-Frobenius matrix is from a homeomorphism on a given surface, actually affects the possible values of entropy (the logarithm of the dilatation).

There are several known results relevant to this question of the minimum dilatation of pseudo-Anosov homeomorphisms. Penner [20] gives a lower bound $2^{1 /(12 g-12+4 n)}$ for the dilatations on $F_{g, n}$ a genus- $g$ surface with $n$ punctures. In [20, 2, 7] pseudo-Anosov homeomorphisms on $F_{g, 0}$ with small dilatations are constructed showing that the minimum dilatation on $F_{g, 0}$ converges to 1 as the genus $g$ increases. Fehrenbach and Los 11] compute a lower bound $(1+\sqrt{2})^{1 / n}$ for the dilatations of pseudo-Anosov disk homeomorphisms (braids) which permute the punctures in one cycle. In [23] a lower bound $2+\sqrt{5}$ for the dilatations of pseudo-Anosov pure braids is given. A pseudo-Anosov disk homeomorphism is represented by a transitive Markov tree map preserving the end point set of the tree with the same topological entropy. Baldwin [1] gives a lower bound $\log 3$ for the topological entropy of transitive Markov tree maps fixing each end point.

The exact values of the minimum dilatations are known only for few simple cases. Zhirov [28] shows that if a pseudo-Anosov homeomorphism on $F_{2,0}$ has an orientable invariant 
foliation, then its dilatation is not less than the largest zero $\lambda_{5}$ of $x^{4}-x^{3}-x^{2}-x+1$, and gives an example of a pseudo-Anosov homeomorphism realizing the dilatation $\lambda_{5}$.

The pseudo-Anosov 3-braid $\sigma_{2} \sigma_{1}^{-1}$ is shown to be the minimum in the forcing partial order among pseudo-Anosov 3-braids by Matsuoka [17] and Handel [13, hence it attains the minimum dilatation. The pseudo-Anosov 4-braid $\sigma_{3} \sigma_{2} \sigma_{1}^{-1}$ is claimed in [24] to have the minimum dilatation, but the proof given in [24] unfortunately contains an error.

Outline. In this paper we prove the following theorem giving at the same time a corrected proof of the minimality of the dilatation of $\sigma_{3} \sigma_{2} \sigma_{1}^{-1} \in B_{4}$.

Theorem 3.9. The 5-braid $\sigma_{1} \sigma_{2} \sigma_{3} \sigma_{4} \sigma_{1} \sigma_{2}$ attains the minimum dilatation of pseudo-Anosov 5-braids.

The dilatation of a pseudo-Anosov braid is invariant under several operations such as conjugation, composing with a full twist, taking inverse, and taking reverse. It turns out that for braid indices 3 to 5 , the pseudo-Anosov braids realizing the minimum dilatations are essentially unique, modulo the aforementioned operations. This could be just a coincidence. It would be a good surprise if some uniqueness property can be proved for the minimum-dilatation pseudo-Anosov braids.

The two main ingredients of the proof of Theorem 3.9] are the construction of folding automata for generating candidate pseudo-Anosov braids for the minimum dilatation, and the following lemma for bounding the word lengths of the candidate braids.

Lemma 3.1. If $M$ is a Perron-Frobenius matrix of dimension $n$ with $\lambda>1$ its largest eigenvalue, then

$$
\lambda^{n} \geq|M|-n+1
$$

where $|M|$ denotes the sum of entries of $M$.

This lemma improves on [19, Theorem 6] and replaces erroneous Lemma 3,4 of [24.

Given a pseudo-Anosov homeomorphism $f:\left(F,\left\{p_{i}\right\}\right) \rightarrow\left(F,\left\{p_{i}\right\}\right)$ on a surface $F$ with punctures $p_{i}$ with negative Euler characteristic $\chi\left(F-\left\{p_{i}\right\}\right)<0$, there exists a train track representative of $f$. There exists an invariant train track $\tau \subset F-\left\{p_{i}\right\}$ which carries $f(\tau)$. In particular there is a splitting sequence

$$
\tau=\tau_{0} \succ \tau_{1} \succ \cdots \succ \tau_{k}=f(\tau)
$$

from $\tau$ to $f(\tau)$, where $\tau_{j} \succ \tau_{j+1}$ is an elementary splitting move.

By observing that there are only finitely many diffeomorphism types of the pair ( $F-$ $\left.\left\{p_{i}\right\}, \tau_{j}\right)$, one can effectively construct a splitting automaton, which is a finite graph with train tracks as its vertices and with splitting moves as its arrows. 
The existence of the train track representative, in particular of the splitting sequence, implies that every pseudo-Anosov homeomorphism appears, up to conjugacy, as a closed path in some splitting automata (see [18]). Papadopoulos and Penner [19, Theorem 6] also gave a lower bound for the dilatation in terms of word length in automata.

In this paper we use folding automata as in [24], which are finite graphs with embedded train tracks as vertices and with elementary folding maps as arrows. An elementary folding map is an inverse of a splitting move.

If we are given an upper bound for the word length in terms of the dilatation, then on a fixed folding automaton, the search for the minimum dilatation in the automaton reduces to checking for finitely many closed paths.

Lemma 3.1. which is an improvement of [19, Theorem 6], not only gives an upper bound of the word lengths of mapping classes with dilatation bounded by a fixed number, but also trims out many branches which appear in the course of search in a big tree, namely the set of paths with bounded length. In fact Lemma 3.1implies that it suffices to consider only such paths whose any subpath has a transition matrix with bounded norm.

For the minimum dilatation of 5-braids, the previously mentioned restriction on paths by transition matrix norm and another restriction by Lemma 3.3 significantly reduce the number of candidate braids making the computation feasible.

We think that the same method for computing the minimum dilatation would still work for a few more simple cases like on a genus-2 closed surface, although it would involve more complicated computer aided search.

\section{Folding Automata}

Given a pseudo-Anosov homeomorphism $f:\left(F,\left\{p_{i}\right\}\right) \rightarrow\left(F,\left\{p_{i}\right\}\right)$ on a closed surface $F$ with punctures $\left\{p_{i}\right\}$, there exists an invariant train track $\tau \subset F-\left\{p_{i}\right\}$ and $f$ is represented by a train track map $f_{\tau}: \tau \rightarrow \tau$ [18].

A train track $\tau$ is a smooth branched 1-manifold embedded in the surface $F-\left\{p_{i}\right\}$ such that each component of the complement $F-\left\{p_{i}\right\}-\tau$ is either a once punctured $k$-gon for $k \geq 1$ or a non-punctured $k$-gon for $k \geq 3$. The train track $\tau$ is called invariant under $f$ if $f(\tau)$ smoothly collapses onto $\tau$ in $F-\left\{p_{i}\right\}$ inducing a smooth map $f_{\tau}: \tau \rightarrow \tau$, which maps branch points to branch points. In this case one may repeatedly fold (or zip) $f(\tau)$ nearby cusps to obtain a train track isotopic to $\tau$ in $F-\left\{p_{i}\right\}$ (see Figure 3 and [15. Fig. 4, 5]).

Let $f_{\tau}: \tau \rightarrow \tau$ be a train track representative of a pseudo-Anosov homeomorphism $f$. An edge $e$ of $\tau$ is called infinitesimal if it is eventually periodic under $f_{\tau}$, that is, $f_{\tau}^{N+k}(e)=$ $f_{\tau}^{N}(e)$ for some $N, k>0$. An edge of $\tau$ is called expanding if it is not infinitesimal. An 

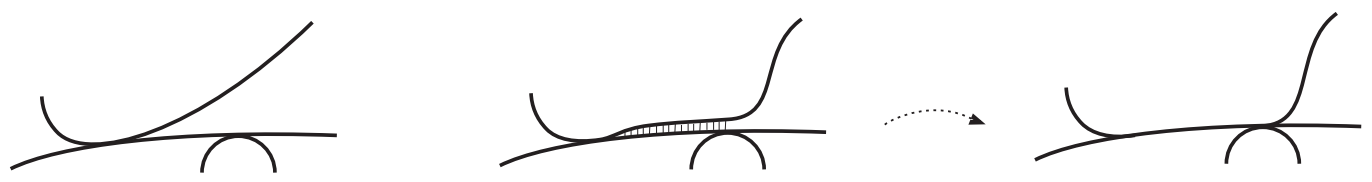

Figure 3. An elementary folding map

expanding edge $e$ actually has a positive length in the sense that $\lim _{N \rightarrow \infty}\left|f_{\tau}^{N}(e)\right| / \lambda_{f}^{N}$ is positive where $|\cdot|$ denotes the word length of a path and $\lambda_{f}=\lambda(f)$ denotes the dilatation factor for $f$.

A graph map is called Markov if it maps vertices to vertices, and is locally injective at points that do not map into vertices. Given a Markov map $g: \tau \rightarrow \tau^{\prime}$, the transition matrix $M_{g}=\left(m_{i j}\right)$ is defined by that the $j$-th edge $\left(e_{j}^{\prime}\right)^{ \pm 1}$ of $\tau^{\prime}$ occurs $m_{i j}$-many times in the path $g\left(e_{i}\right)$, the image of the $i$-th edge of $\tau$. When $\tau^{\prime}=\tau$, the transition matrix is square and considering its spectral radius makes sense.

The spectral radius of $M_{f_{\tau}}$ equals the dilatations factor $\lambda(f)$ for the pseudo-Anosov homeomorphism $f$. Coordinates of the corresponding eigenvectors of $M_{f_{\tau}}$ and its transpose $M_{f_{\tau}}^{T}$ are tangential and transverse measures of edges of $\tau$, which are projectively invariant under $f$.

An elementary folding map $\pi: \tau \rightarrow \tau^{\prime}$ is a smooth Markov map between two train tracks $\tau$ and $\tau^{\prime}$ such that for only one edge $e$ of $\tau$, the image $\pi(e)$ has word length 2 , and the other edges map to paths of length 1 . In other words the transition matrix $M_{\pi}$ is of the form $P+B$ for some permutation matrix $P$ and for some elementary matrix $B$.

When the train tracks are embedded in a surface as in our case of concern, the pairs of edges which are folded should be adjacent in the surface: the two segments of $\tau$ which are identified by the elementary folding map are two sides of an open triangle in $F-\left\{p_{i}\right\}-\tau$ (see Figure 3]).

Proposition 2.1. A train track representative $f_{\tau}: \tau \rightarrow \tau$ of a surface homeomorphism $f:\left(F,\left\{p_{i}\right\}\right) \rightarrow\left(F,\left\{p_{i}\right\}\right)$ admits a folding decomposition as follows:

$$
f_{\tau}=\rho \circ \pi_{k} \circ \cdots \circ \pi_{1}
$$

where $\pi_{j}: \tau_{j} \rightarrow \tau_{j+1}$ are elementary folding maps, $\tau_{1}=\tau_{k+1}=\tau$, and $\rho: \tau \rightarrow \tau$ is an isomorphism induced by a periodic surface homeomorphism $\left(F-\left\{p_{i}\right\}, \tau\right) \rightarrow\left(F-\left\{p_{i}\right\}, \tau\right)$ preserving $\tau$.

Proof. It follows from [25]. See [18, 24] for more details. 
By observing that there are only finitely many possible diffeomorphism types for the pairs $\left(F-\left\{p_{i}\right\}, \tau_{j}\right)$ appearing in the folding decomposition, we can construct folding automata. A folding automaton is a connected directed graph with diffeomorphism types of train tracks as vertices, with elementary folding maps and isomorphisms as arrows. See Figure 7 for a simplified version of a folding automaton. The train tracks in Figure 7 admit no non-trivial isomorphisms, that is, if $h:\left(D_{5}, \tau\right) \rightarrow\left(D_{5}, \tau\right)$ is an orientation preserving diffeomorphism fixing $\tau$ in the automaton, then $h$ is isotopic to the identity map. So in Figure 7 there are no arrows corresponding to isomorphisms.

Corollary 2.2. All train track representatives of pseudo-Anosov homeomorphisms are represented by closed oriented paths in folding automata.

To each closed path based at a train track $\tau$ in a folding automaton, associated is a train track representative $f_{\tau}: \tau \rightarrow \tau$ of some homeomorphism $f:\left(F,\left\{p_{i}\right\}\right) \rightarrow\left(F,\left\{p_{i}\right\}\right)$. The disk homeomorphism $f$ is pseudo-Anosov if and only if the transition matrix $M_{f_{\tau}}$ is Perron-Frobenius (also called primitive) modulo infinitesimal edges: for some $N>0$, the power $M_{f_{\tau}}^{N}$ is strictly positive in the block of expanding edges. To find out whether $M$ is Perron-Frobenius, it suffices by [14, 27], [21, Theorem 2.8 on p.52] to check if $M^{n^{2}-2 n+2}$ has all non-zero entries where $n$ is the dimension of the matrix $M$.

Now we discuss simplifying the train track maps so that we can restrict to simplified folding automata. If the pseudo-Anosov homeomorphism $f$ fixes a distinguished puncture $p_{0}$ : $f\left(p_{0}\right)=p_{0}$ (for instance when $f$ is from a disk homeomorphism and $p_{0}$ is the boundary puncture), we can give a restriction to the train track map $f_{\tau}: \tau \rightarrow \tau$, thereby reducing the size of the folding automata needed in our computation.

We first assume that only the component of $F-\tau$ containing $p_{0}$ has expanding edges on its sides: the other components of $F-\tau$ not containing $p_{0}$ are bounded only by infinitesimal edges. If one is given a train track representative $f_{\tau}: \tau \rightarrow \tau$ not satisfying this assumption, he can apply a splitting operation [5, Section 5] nearby $p_{0}$ (when $p_{0}$ is enclosed only by infinitesimal edges) then apply a sequence of folding operations [4, p.15] [16. Section 2.2] nearby other punctures $p_{i}, i \neq 0$, until all the components of the train track complement not containing $p_{0}$ shrink to be infinitesimal, to obtain a new train track representative satisfying the assumption [4, Proposition 3.3].

Applying some more folding operations (see Figure 4), we can also remove any cusp occurring between an expanding edge and an infinitesimal edge.

We assume that cusps occur only at corners of infinitesimal multigons. If one is given a train track representative with a cusp incident only to expanding edges, not satisfying 


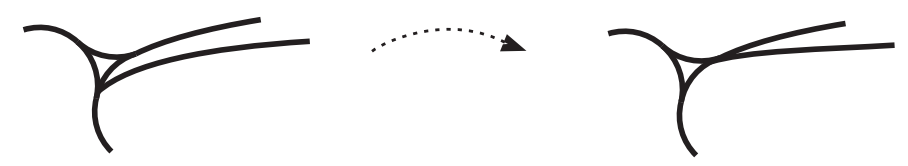

FiguRE 4. A folding operation which absorbs an infinitesimal edge

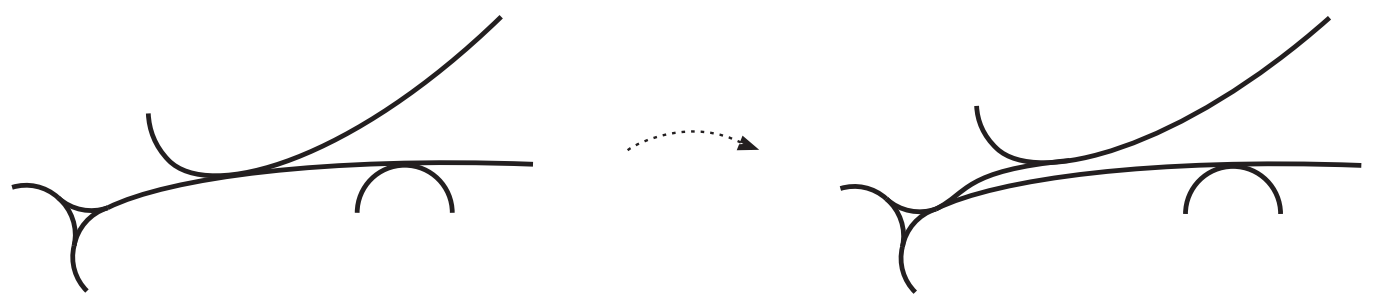

FigURE 5. A splitting operation which shifts a cusp incident only to expanding edges
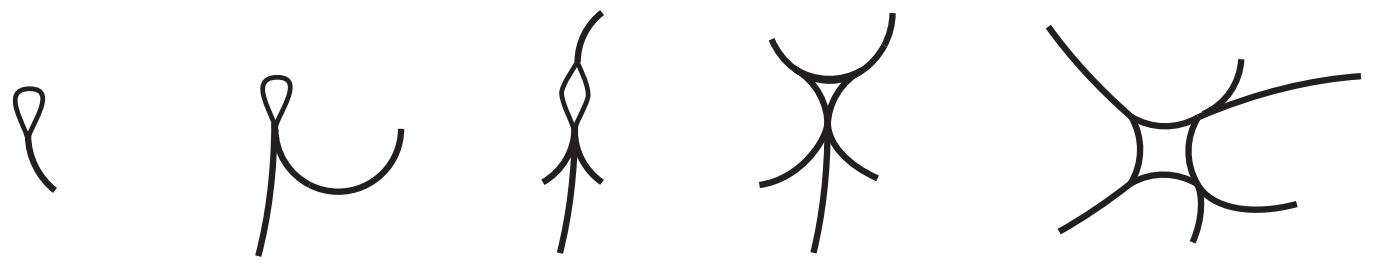

FigURE 6. Local models for train tracks in simplified folding automata

this assumption, he can apply a splitting operation at the cusp until the cusp hits an infinitesimal multigon (see Figure 51).

Therefore a pseudo-Anosov braid has an invariant train track which is locally modeled by infinitesimal $k$-gons to which expanding edges are joined (possibly) forming cusps only between expanding edges (see Figure 6).

In this paper we use simplified versions of folding automata, of which train tracks satisfy the previously given conditions, and each arrow is either an isomorphism or a composite of two elementary folding maps by which one expanding edge and one infinitesimal edge is absorbed into another expanding edge. It is not hard to see that simplified folding automata also generate all the conjugacy classes of pseudo-Anosov homeomorphisms.

In this paper our subject of interest is pseudo-Anosov homeomorphisms on a 5-times punctured disk $D_{5}$, or equivalently on a 6 -times punctured sphere $F_{0,6}$ with a distinguished boundary puncture. 


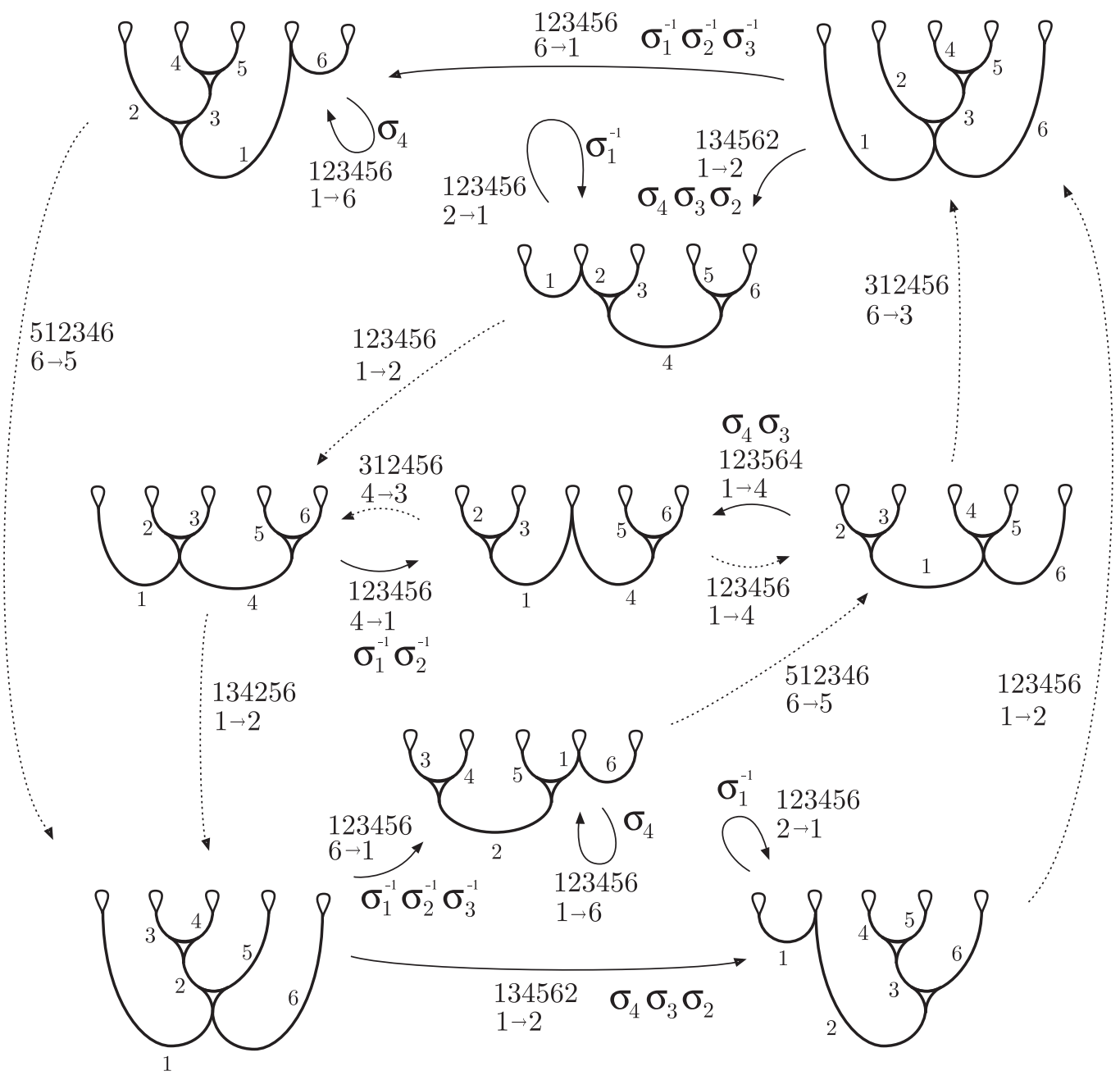

Figure 7. A folding automaton for pseudo-Anosov 5-braids with two nonpunctured 3-prong singularities

We explain how to read Figure [7, which depicts a simplified version of a folding automaton. Each train track is embedded in a 5-times punctured disk, with each puncture enclosed by an infinitesimal monogon. Each embedding is chosen arbitrarily, and only the orientation preserving diffeomorphism types of embedded train tracks count.

An arrow is a composite of two elementary folding maps, one involving an infinitesimal edge and another involving only expanding edges. We ignore the infinitesimal edges in computing the transition matrix because the occurrences of infinitesimal edges do not affect the resulting dilatation factor. 
An arrow is drawn dashed if it induces a homeomorphism isotopic to identity, and it is drawn by a solid line otherwise. Note that a folding map $\pi: \tau \rightarrow \tau^{\prime}$ determines a disk homeomorphism $f: D_{5} \rightarrow D_{5}$ up to isotopy when the embeddings $\tau \rightarrow D_{5}$ and $\tau^{\prime} \rightarrow D_{5}$ of the two train tracks are fixed. In particular $\tau^{\prime} \succ f(\tau)$, that is, $f(\tau)$ folds to be $\tau^{\prime}$ inducing the folding map $\pi$. To each solid arrow, a braid word is assigned representing the associated disk homeomorphism.

Edges of a train track are numbered by $\{1,2, \cdots, 6\}$ in such a way that in the peripheral word running clockwise from a cusp, new edges appear in an increasing order. This naming of edges amounts to fixing a groupoid homomorphism from paths in the automaton to transition matrices, that is, for two paths $\gamma$ and $\delta, M(\gamma \cdot \delta)=M(\gamma) M(\delta)$ if $\gamma$ ends at the starting vertex of $\delta$, where $M(\gamma)$ denotes the transition matrix for $\gamma$.

Each arrow is associated with a permutation $i_{1} i_{2} i_{3} i_{4} i_{5} i_{6}$ and a rule $m \rightarrow n$, meaning that under the elementary folding map, the edge $j$ maps to $i_{j}$ for $j \neq m$, and $m$ maps to $i_{m} \cdot n$. (Here we concern only the transition matrix so that the direction of edges and the order of concatenation are irrelevant.)

Given an adjacent pair $\left(e_{1}, e_{2}\right)$ of edges with a cusp between them, there are two possible folding maps: one under which the image of $e_{1}$ overpasses that of $e_{2}$ and another vice versa. Therefore from each train track in Figure 7 two arrows of elementary folding maps emanate. Likewise two arrows are headed for each train track, because at each cusp there are two different elementary splittings possible.

\section{SeArCh FOR the minimum Dilatation}

In this section we prove that the largest zero $\lambda_{5}$ of $x^{4}-x^{3}-x^{2}-x+1$ is indeed the minimum dilatation for pseudo-Anosov 5-braids.

The problem for the minimum dilatation reduces to a search in a finite set of closed paths in folding automata because by [19, Theorem 6] or by Lemma 3.1 the dilatation grows as the norm of the transition matrix grows, and there are only finitely many closed paths whose transition matrices have norm bounded by a given number. For instance if a closed path in folding automata has length $N$, then its associated transition matrix has norm at least $N$.

We first restate and prove the lemma in Section 1.

Lemma 3.1. If $M$ is a Perron-Frobenius matrix of dimension $n$ with $\lambda>1$ its largest eigenvalue, then

$$
\lambda^{n} \geq|M|-n+1
$$

where $|M|$ denotes the sum of entries of $M$. 
Proof. Let $M=\left(m_{i j}\right)$ and let $\left(v_{i}\right)$ be the eigenvector given by the equation

$$
\lambda v_{i}=\sum_{j=1}^{n} m_{i j} v_{j}
$$

for $v_{i}>0,1 \leq i \leq n$.

The matrix $M$ is the transition matrix of a graph $G$ with vertex set $V(G)=\{1,2, \ldots, n\}$ such that the number of oriented edges from $i$ to $j$ equals $m_{i j}$. Let $M^{n}=\left(k_{i j}\right)$. The number of paths with length $n$ from $i$ to $j$, equals $k_{i j}$. For each pair $(i, j)$ of vertices there exists an oriented path from $i$ to $j$ since $M$ is Perron-Frobenius.

Note that $\left(v_{i}\right)$ is also the eigenvector of $M^{n}$ with eigenvalue $\lambda^{n}$. Choose $v_{p}=\min _{i} v_{i}$ the smallest coordinate of $\left(v_{i}\right)$.

$$
\begin{aligned}
\lambda^{n} v_{p} & =\sum_{j} k_{p j} v_{j} \\
& \geq\left(\sum_{j} k_{p j}\right) v_{p} \quad \text { since } v_{j} \geq v_{p}
\end{aligned}
$$

The inequality $\lambda^{n} \geq \sum_{j} k_{p j}$ reads that $\lambda^{n}$ is not less than the number of length- $n$ paths from the vertex $p$ of $G$.

Take a maximal positive tree $T \subset G$ rooted at $p$ : each vertex of $G$ is connected to $p$ by a unique oriented path in $T$. As $T$ is maximal, $|V(T)|=|V(G)|=n$ so that the number of edges of $T$ is $|E(T)|=n-1$.

If an oriented path $\gamma$ from $p$ has length $n$, it must digress from $T$ at some point. Define $a(\gamma)=e \in E(G)-E(T)$ to be the first edge not in $E(T)$ of $\gamma$. This defines a function

$$
a \text { : }\{\text { length- } n \text { paths from } p \text { in } G\} \rightarrow E(G)-E(T) .
$$

Clearly $a$ is a surjection since the tail of each edge in $E(G)-E(T)$ is connected to $p$ by a path in $T$ with length at most $n-1$. Therefore

$$
\lambda^{n} \geq \sum_{j} k_{p j} \geq|E(G)-E(T)|=|M|-(n-1) .
$$

Theorem 3.2 (24). If a pseudo-Anosov 4-braid has an invariant foliation with one nonpunctured 3-prong singularity, then its dilatation is not less than $\lambda_{4} \approx 2.29663$, the largest zero of $x^{4}-2 x^{3}-2 x+1$.

Proof. Let $\beta \in B_{4}$ be a pseudo-Anosov 4-braid in the theorem. Up to conjugacy and multiplication by central elements, $\beta$ appears as a closed path $\gamma$ in the folding automaton in Figure 8 


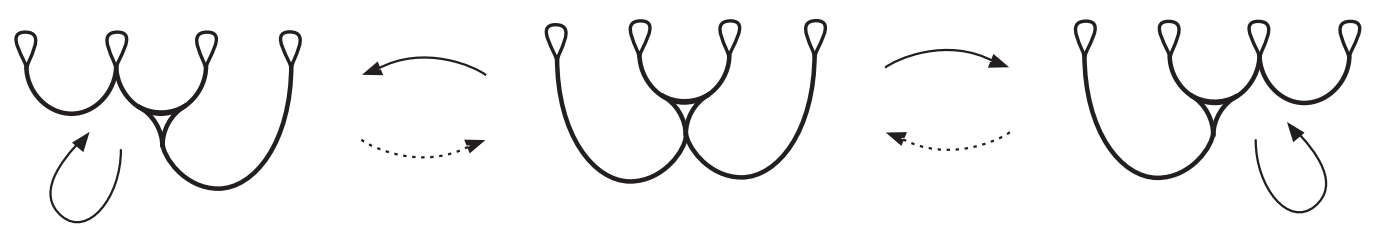

Figure 8. A simplified folding automaton for pseudo-Anosov 4-braids with a 3-prong singularity

If $\lambda(\beta)<2.3$, then by Lemma 3.1 we have a bound $31>2.3^{4}+4-1 \geq\left|M_{\gamma}\right|$ for the norm of its transition matrix $M_{\gamma}$.

By a computer aided search [22] in the finite set of paths $\gamma$ with $\left|M_{\gamma}\right|<31$, we conclude that up to conjugacy, multiplication by central elements, and taking inverse, the braid $\sigma_{3} \sigma_{2} \sigma_{1}^{-1}$ is the only pseudo-Anosov 4 -braid with dilatation less than 2.3. It can be easily checked that $\lambda\left(\sigma_{3} \sigma_{2} \sigma_{1}^{-1}\right)=\lambda_{4}$.

We say two matrices have the same pattern if they have zero entries and positive entries in the same positions. We write $M \geq M^{\prime}$ for $M=\left(m_{i j}\right)$ and $M^{\prime}=\left(m_{i j}^{\prime}\right)$ if $m_{i j} \geq m_{i j}^{\prime}$ for all $i, j$.

In the following lemma we ignore the parts of transition matrices arising from infinitesimal edges, so that for a closed path to represent a pseudo-Anosov homeomorphism implies for its transition matrix to be Perron-Frobenius.

Lemma 3.3. Let $\gamma$ be a closed path in a folding automaton. Let $N, k>0$ be numbers such that the transition matrices $M\left(\gamma^{N+i+k}\right)$ and $M\left(\gamma^{N+i}\right)$ have the same pattern and $M\left(\gamma^{N+i+k}\right) \geq M\left(\gamma^{N+i}\right)$ for any $i \geq 0$. Then a closed path of the form $\alpha \cdot \gamma^{N+i+k} \cdot \delta$ in the folding automaton represents a pseudo-Anosov homeomorphism if and only if $\alpha \cdot \gamma^{N+i} \cdot \delta$ does. Furthermore in this case we have an inequality

$$
\lambda\left(\alpha \cdot \gamma^{N+i} \cdot \delta\right) \leq \lambda\left(\alpha \cdot \gamma^{N+i+k} \cdot \delta\right)
$$

between their dilatation factors.

Proof. It suffices to prove the lemma for $\delta \cdot \alpha \cdot \gamma^{N+i+k}$ and $\delta \cdot \alpha \cdot \gamma^{N+i}$ since conjugation does not affect dilatation factor or being pseudo-Anosov.

Since $M\left(\gamma^{N+i+k}\right)$ and $M\left(\gamma^{N+i}\right)$ have the same pattern, $M(\delta \cdot \alpha) M\left(\gamma^{N+i+k}\right)$ and $M(\delta$. $\alpha) M\left(\gamma^{N+i}\right)$ also have the same pattern. In particular one is Perron-Frobenius if and only if so is the other, which proves the first claim of the lemma.

From $M\left(\gamma^{N+i}\right) \leq M\left(\gamma^{N+i+k}\right)$, we have

$$
M\left(\delta \cdot \alpha \cdot \gamma^{N+i}\right) \leq M\left(\delta \cdot \alpha \cdot \gamma^{N+i+k}\right),
$$


which by [21, Theorem 1.1 (e)] implies the inequality of the lemma.

Remark 3.4. Let $\gamma, N, k$ be given as in Lemma 3.3. Then the lemma implies that when we search just for the minimum dilatation factor for pseudo-Anosov homeomorphisms, it suffices to search in the set of paths that do not contain $\gamma^{N+k}$ as a subpath.

In the search in the automaton in Figure [7, we exclude paths containing several closed paths, for example $\left(\begin{array}{c}123564 \\ 1 \rightarrow 4\end{array} \stackrel{1}{1 \rightarrow 4}_{1 \rightarrow 4}^{2346}\right)^{6},\left(\begin{array}{c}123456 \\ 4 \rightarrow 1\end{array} \cdot \begin{array}{c}312456 \\ 4 \rightarrow 3\end{array}\right)^{6}$, and second iterates of length 1 loops. This reduces the size of the set of candidate braids for minimum dilatation to the extent that the computation in the proof of Theorem 3.5 becomes possible on a personal computer.

Theorem 3.5. If a pseudo-Anosov 5-braid has an invariant foliation with two non-punctured 3-prong singularities, then its dilatation is not less than the largest zero $\approx 2.01536$ of $x^{6}-x^{5}-4 x^{3}-x+1$.

Proof. It is easy to check that there are only nine different diffeomorphism types of train tracks in $D_{5}$, locally modeled by infinitesimal multigons with outgoing expanding-edge legs as in Figure 6, By computing the elementary folding maps among them (more precisely composites of two elementary folding maps, one of them involving an infinitesimal edge), we have a folding automaton depicted in Figure 7.

By a computer aided search [22] in the set of paths $\gamma$ with $\left|M_{\gamma}\right| \leq 2.02^{6}+5<73$, we conclude that up to conjugacy and multiplication by central elements, $\sigma_{4} \sigma_{3} \sigma_{1}^{-1} \sigma_{2}^{-1}$ with dilatation $\approx 2.01536$ is the only such pseudo-Anosov 5-braid with dilatation less than 2.02 .

Lemma 3.6. If a pseudo-Anosov 5-braid has an invariant foliation with a non-punctured 4-prong singularity, then its dilatation is not less than the largest zero $\approx 2.15372$ of $x^{4}-$ $3 x^{3}+3 x^{2}-3 x+1$.

Proof. The folding automaton for this case is similar to one in Figure 8 . As in the proof of Theorem 3.2, a computer aided search [22] in the set of closed paths up to length $56>2.2^{5}+(5-1)$ shows that the largest zero of $x^{4}-3 x^{3}+3 x^{2}-3 x+1$ is the minimum dilatation factor in the automaton, and it is achieved by $\sigma_{4} \sigma_{3} \sigma_{2} \sigma_{1}^{-1}$.

Lemma 3.7. If a pseudo-Anosov 5-braid has an invariant foliation with a punctured 3prong singularity, then its dilatation is not less than $\lambda_{5}$, the largest zero of $x^{4}-x^{3}-x^{2}-x+1$.

Proof. Let $f: F_{0,6} \rightarrow F_{0,6}$ be a pseudo-Anosov homeomorphism with an invariant foliation $\mathcal{F}$. If the invariant measured foliation $\mathcal{F}$ on a 6 -times punctured sphere has a punctured 3-prong singularity, then it has five other punctured 1-prong singularities and no more. 

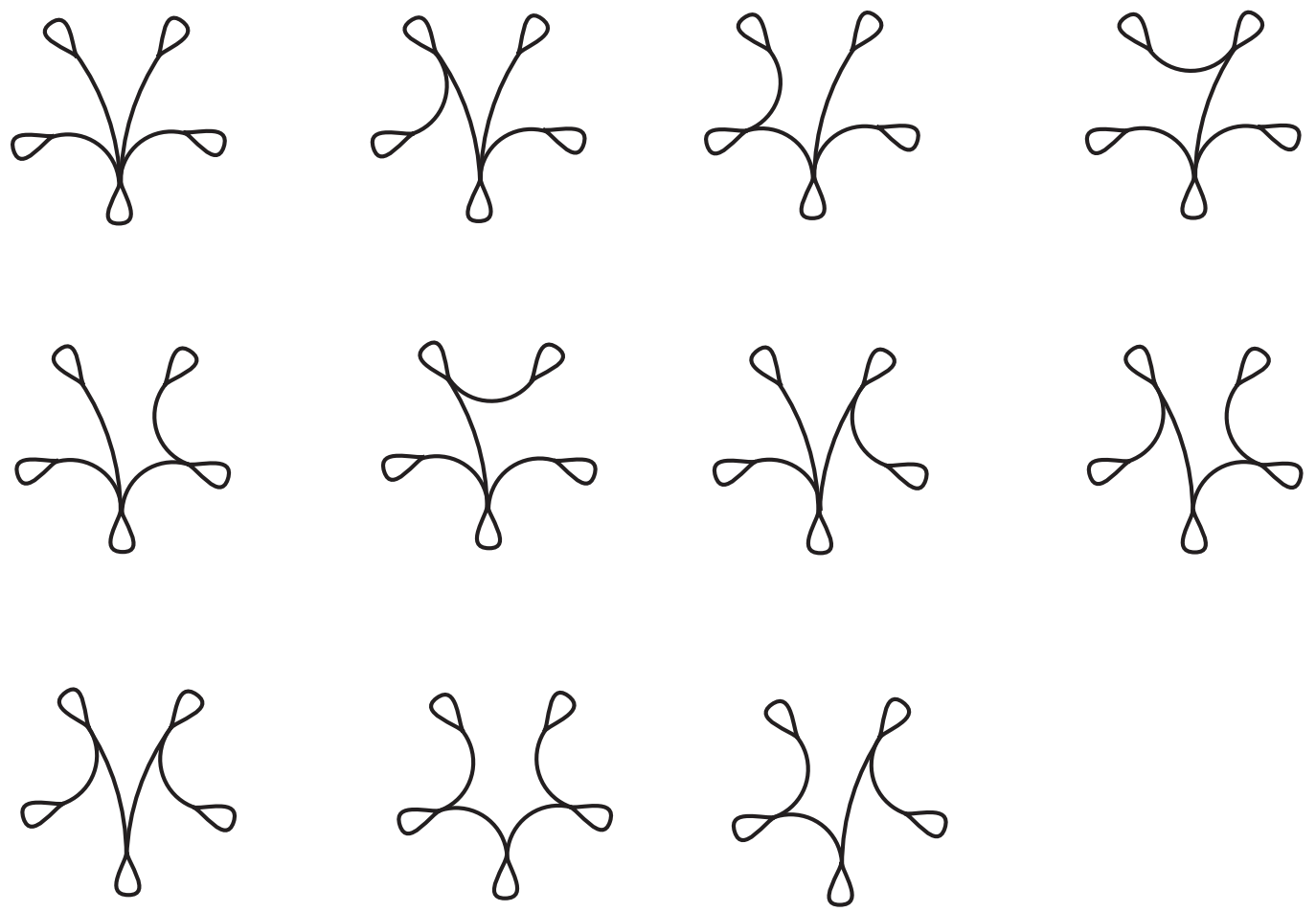

Figure 9. Train tracks for pseudo-Anosov 5-braids with a 3-pronged boundary puncture

We can assume that the punctured 3-prong singularity is the boundary puncture since it should be fixed by the homeomorphism $f$. Now we use the folding automaton that generates such pseudo-Anosov braids with three prongs at the boundary puncture.

There are eleven diffeomorphism types of train tracks to consider for this case (see Figure 9). There are fifty arrows in the automaton, which are too many to be drawn in a figure in this paper. See [22] for details.

By the same kind of computer aided search as before, in the set of closed paths in the folding automaton up to length $12 \geq\left(\lambda_{5}\right)^{4}+(4-1)$, we conclude that $\lambda_{5}$ is the minimum dilatation factor for pseudo-Anosov braids in this automaton.

The dilatation is achieved by $\sigma_{1} \sigma_{2} \sigma_{3} \sigma_{4} \sigma_{1} \sigma_{2}$.

Remark 3.8. In [28], $\lambda_{5}$ is proved to be the minimum dilatation factor for a pseudo-Anosov homeomorphism with an orientable invariant foliation on a closed genus- 2 surface. The proof in 28] seems to have a gap. To complete the proof one needs to show that the golden ratio $(1+\sqrt{5}) / 2 \approx 1.61803$ the largest zero of $x^{4}-3 x^{2}+1$, cannot be a dilatation factor for such a pseudo-Anosov homeomorphism. In [12, it is proved that such a pseudo-Anosov 
homeomorphism with quadratic dilatation factor is a lift of an Anosov homeomorphism via a branched covering. Lemma 3.6 and Lemma 3.7 follow from [28] by taking double covers branched at odd-prong singularities.

By collecting all the results, we conclude this paper with a proof of the main theorem.

Theorem 3.9. The 5-braid $\sigma_{1} \sigma_{2} \sigma_{3} \sigma_{4} \sigma_{1} \sigma_{2}$ attains the minimum dilatation of pseudo-Anosov 5-braids.

Proof. Let $f: F_{0,6} \rightarrow F_{0,6}$ be a pseudo-Anosov homeomorphism on a 6 -times punctured sphere with a punctured point fixed by $f$. Let $\mathcal{F}$ be its invariant measured foliation. Since $\mathcal{F}$ has exactly six punctures, the formula $2=\chi\left(F_{0,0}\right)=\sum_{k}(1-k / 2) n_{k}$ where $n_{k}$ denotes the number of $k$-prong singularities, says no singularity of $\mathcal{F}$ can have more than four prongs.

The list of possible types of $\mathcal{F}$ according to its singularity type is:

(1) six punctured 1-prong singularities and one non-punctured 4-prong singularity: $n_{1}=6, n_{4}=1$

(2) six punctured 1-prong singularities and two non-punctured 3-prong singularities: $n_{1}=6, n_{3}=2$

(3) five punctured 1-prong singularities and one punctured 3-prong singularity: $n_{1}=$ $5, n_{3}=1$

(4) five punctured 1-prong singularities, one punctured 2-prong singularity, and one non-punctured 3-prong singularities: $n_{1}=5, n_{2}=1, n_{3}=1$

(5) four punctured 1-prong singularities and two punctured 2-prong singularities: $n_{1}=$ $4, n_{2}=2$

The case (5) is, by capping-off 2-prong singularity punctures, of a pseudo-Anosov homeomorphism on a four-times punctured sphere, which lifts to an Anosov homeomorphism on a torus via branched double covering. Therefore in this case $\lambda(f) \geq(3+\sqrt{5}) / 2>\lambda_{5}$.

The case (4) reduces to the case (3) by capping-off the punctured 2-prong singularity and puncturing at the 3-prong singularity.

For the cases (11) and (3) we have $\lambda(f) \geq \lambda_{5}$ by Lemma 3.6 and Lemma 3.7] or by Remark 3.8 .

Finally the case (2) is covered by Theorem 3.5, so that we have $\lambda(f)>2.01>\lambda_{5}$. In fact this is the only part of the proof which actually requires a computer aided search if one uses Zhirov's result [28].

Collecting all of these we conclude that $\lambda(f) \geq \lambda_{5} \approx 1.72208$. It is easily checked that $\beta=\sigma_{1} \sigma_{2} \sigma_{3} \sigma_{4} \sigma_{1} \sigma_{2}$ realizes this dilatation $\lambda(\beta)=\lambda_{5}$. 


\section{IMPLEMENTATION}

To search for the minimum-dilatation pseudo-Anosov homeomorphism on a given surface, we first need to generate a collection of folding automata. For 5-braids it is possible to build the necessary folding automata manually. On surfaces with more punctures and greater genus, we also need a computer program genauto to generate the folding automata. This paper will not cover the details of its implementation. The following is a pseudo-code for genauto.

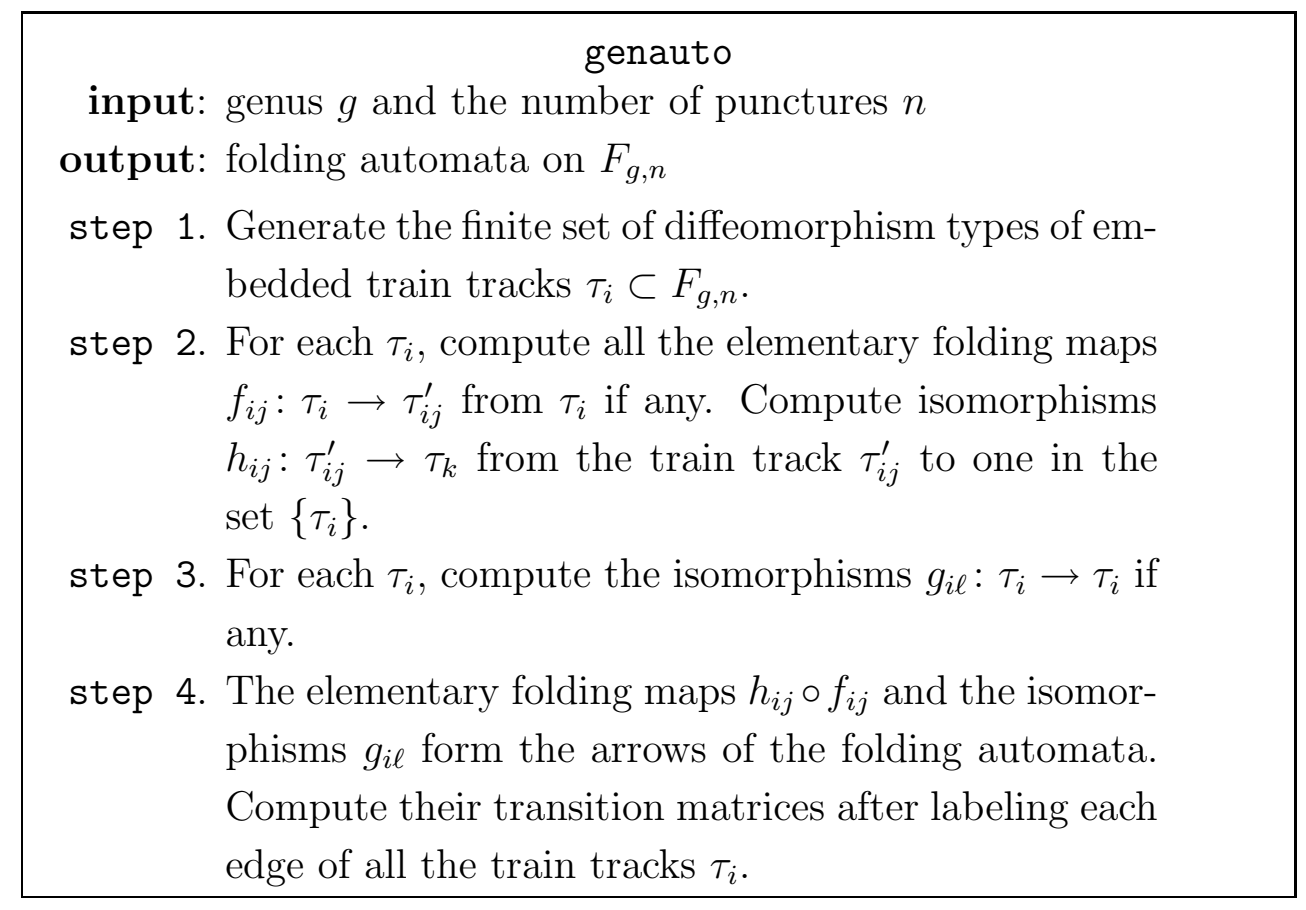

Note that for step 1 one needs to solve the isomorphism problem for embedded train tracks. Once step 1 is done, implementing the other steps is more straightforward.

By running genauto, we obtain the folding automata as a collection of connected directed graph with each arrow labeled by a transition matrix. The goal is to enumerate in the folding automata all the closed paths representing pseudo-Anosov mapping classes with an upper bound for the dilatation.

In this paper we deal with 5-braids using simplified folding automata. We ignore infinitesimal edges when computing transition matrices. Therefore a closed path in a folding automaton represents a pseudo-Anosov braid if and only if its associated transition matrix is Perron-Frobenius.

The following is a pseudo-code for our program fbrmin. See 22 for details. 


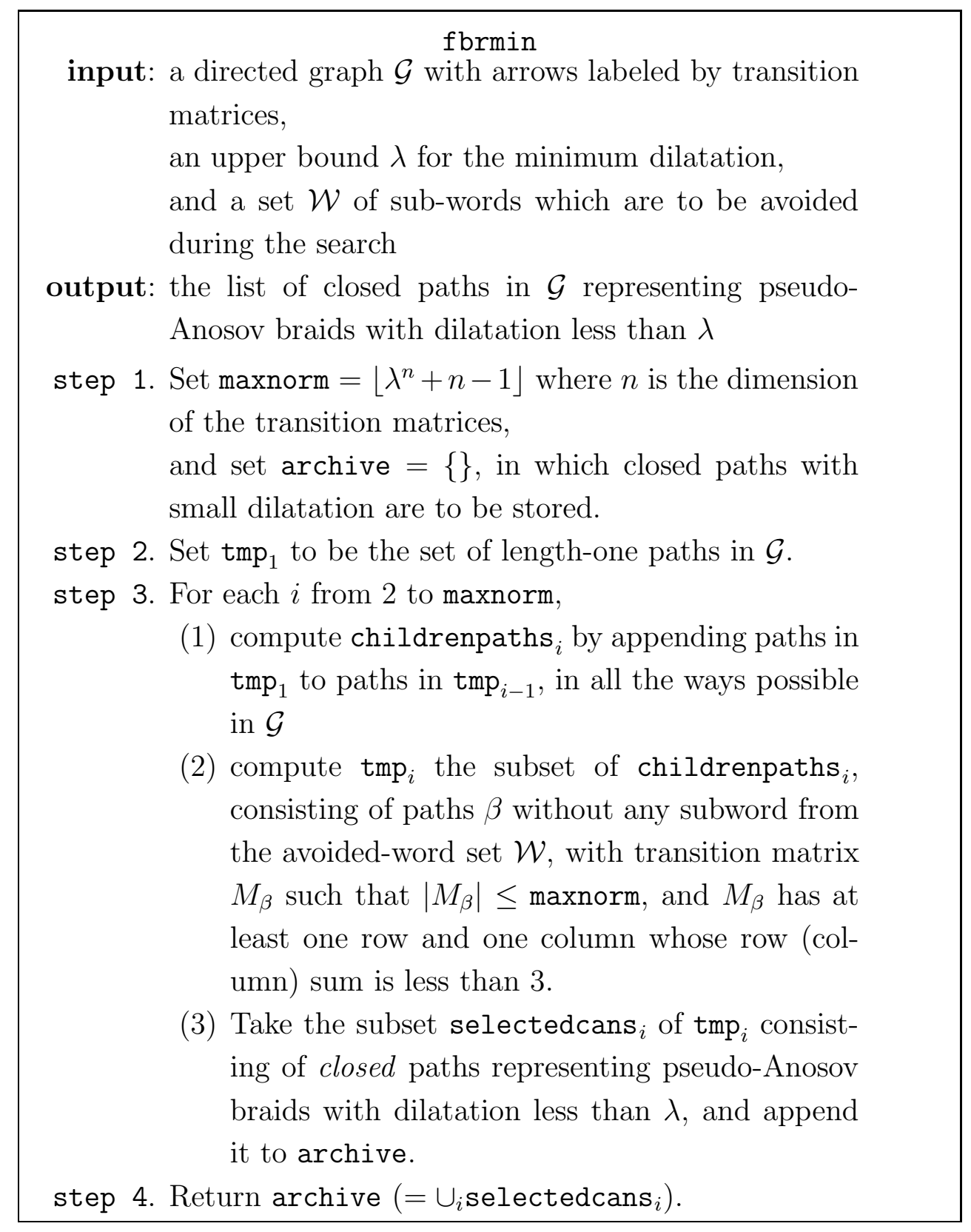

In step 3 (2), we use Lemma 3.1, 3.3 to trim out much of unnecessary computation (see Remark 3.4).

When the row sums of a transition matrix $M_{\beta}$ all exceed 3, then the spectral radius of $M_{\beta}$ is greater than 3. In this case the same holds for every transition matrix of the form $M_{\beta \cdot \gamma}=M_{\beta} M_{\gamma}$ since $M_{\gamma} \geq P$ for some permutation matrix $P$. Therefore as we are looking for transition matrices with spectral radius less than 3 , we can safely remove such paths $\beta \cdot \gamma$ from our consideration, as done in step $3(2)$. 
For computational aspects, the proof of Theorem 3.5 using the automaton in Figure 7 is the main part which consumes most time and memory. On a $2.40 \mathrm{GHz}$ machine, it took 1000 seconds of time and 150 mega-bytes of memory. During the search roughly 85000 many matrices were actually tested for its largest eigenvalue.

We do not know how far the same kind of computation would work for more complicated surfaces. We expect that at least the case for 6-braids, hence for genus-2 closed surface, can be done on a personal computer without too much difficulty.

\section{REFERENCES}

[1] S. Baldwin. Entropy estimates for transitive maps on trees. Topology, 40(3):551-569, 2001.

[2] M. Bauer. An upper bound for the least dilatation. Trans. Amer. Math. Soc., 330(1):361-370, 1992.

[3] L. Bers. An extremal problem for quasiconformal mappings and a theorem by Thurston. Acta Math., 141(1-2):73-98, 1978.

[4] M. Bestvina and M. Handel. Train tracks and automorphisms of free groups. Ann. of Math. (2), 135(1):1-51, 1992.

[5] M. Bestvina and M. Handel. Train-tracks for surface homeomorphisms. Topology, 34(1):109-140, 1995.

[6] J. S. Birman and M. E. Kidwell. Fixed points of pseudo-Anosov diffeomorphisms of surfaces. Adv. in Math., 46(2):217-220, 1982.

[7] P. Brinkmann. A note on pseudo-Anosov maps with small growth rate. Experiment. Math., 13(1):49$53,2004$.

[8] C. Cao and G. R. Meyerhoff. The orientable cusped hyperbolic 3-manifolds of minimum volume. Invent. Math., 146(3):451-478, 2001.

[9] G. Daskalopoulos and R. Wentworth. Classification of Weil-Petersson isometries. Amer. J. Math., 125(4):941-975, 2003.

[10] A. Fathi, F. Laudenbach, and V. Poénaru. Travaux de Thurston sur les surfaces, volume 66 of Astérisque. Société Mathématique de France, Paris, 1979. Séminaire Orsay, With an English summary.

[11] J. Fehrenbach and J. Los. Une minoration de l'entropie topologique des difféomorphismes du disque. J. London Math. Soc. (2), 60(3):912-924, 1999.

[12] J. Franks and E. Rykken. Pseudo-Anosov homeomorphisms with quadratic expansion. Proc. Amer. Math. Soc., 127(7):2183-2192, 1999.

[13] M. Handel. The forcing partial order on the three times punctured disk. Ergodic Theory Dynam. Systems, 17(3):593-610, 1997.

[14] J. C. Holladay and R. S. Varga. On powers of non-negative matrices. Proc. Amer. Math. Soc., 9:631634, 1958.

[15] R. D. Kleinberg and W. W. Menasco. Train tracks and zipping sequences for pseudo-Anosov braids. Chaos Solitons Fractals, 9(4-5):793-809, 1998. Knot theory and its applications.

[16] J. E. Los. On the conjugacy problem for automorphisms of free groups. Topology, 35(3):779-808, 1996. With an addendum by the author. 
[17] T. Matsuoka. Braids of periodic points and a 2-dimensional analogue of Sharkovskiı̌'s ordering. Sūrikaisekikenkyūsho Kōkyūroku, (574):241-255, 1985. Dynamical systems and nonlinear oscillation phenomena (Japanese) (Kyoto, 1985).

[18] A. Papadopoulos and R. C. Penner. A characterization of pseudo-Anosov foliations. Pacific J. Math., 130(2):359-377, 1987.

[19] A. Papadopoulos and R. C. Penner. Enumerating pseudo-Anosov foliations. Pacific J. Math., 142(1):159-173, 1990.

[20] R. C. Penner. Bounds on least dilatations. Proc. Amer. Math. Soc., 113(2):443-450, 1991.

[21] E. Seneta. Non-negative matrices. Halsted Press [A division of John Wiley \& Sons], New York, 1973. An introduction to theory and applications.

[22] W. T. Song. fbrmin.m: a program for computing the minimum dilatation of pseudo-Anosov 5-braids. 2005. a Mathematica script with commentary, attached to the source file of arXiv:math.GT/0506295.

[23] W. T. Song. Upper and lower bounds for the minimal positive entropy of pure braids. Bull. London Math. Soc., 37(2):224-229, 2005.

[24] W. T. Song, K. H. Ko, and J. E. Los. Entropies of braids. J. Knot Theory Ramifications, 11(4):647666, 2002. Knots 2000 Korea, Vol. 2 (Yongpyong).

[25] J. R. Stallings. Topology of finite graphs. Invent. Math., 71(3):551-565, 1983.

[26] W. P. Thurston. On the geometry and dynamics of diffeomorphisms of surfaces. Bull. Amer. Math. Soc. (N.S.), 19(2):417-431, 1988.

[27] H. Wielandt. Unzerlegbare, nicht negative Matrizen. Math. Z., 52:642-648, 1950.

[28] A. Y. Zhirov. On the minimum dilation of pseudo-Anosov diffeomorphisms of a double torus. Uspekhi Mat. Nauk, 50(1(301)):197-198, 1995.

Department of Mathematics, University of California Santa Barbara, CA 93106

E-mail address: ham@mail.math.ucsb.edu

School of Mathematics, Korea Institute for Advanced Study, 207-43 Cheongnyangni 2-Dong, Dongdaemun-gu, Seoul 130-722, Korea

E-mail address: cape@kias.re.kr 